\title{
Rapid tranquillisation: a global perspective
}

\author{
Pallavi Nadkarni, ${ }^{1}$ Mahesh Jayaram, ${ }^{2}$ Shailesh Nadkarni, ${ }^{3}$ Ranga Rattehalli ${ }^{4}$ \\ and Clive E. Adams ${ }^{5}$
}

${ }^{1}$ Assistant Professor, Department of Psychiatry, Queen's University, Kingston, Ontario, Canada, email nadkarnp@kgh karinet (at the time of the project, she (at the time of the project, she was a ST 4 trainee with Leed Partnerships Foundation NHS Trust)

2Senior Lecturer, Department of Psychiatry, University of of Psychiatry, University of
Melbourne, Australia ${ }^{3}$ Vice-President, National Research Corporation Canada, Markham, Ontario, Canada

${ }^{4}$ Consultant Psychiatrist, Newsam Centre, Seacroft Hospital, Leeds, UK

${ }^{5}$ Professor of Psychiatry, University of Nottingham, UK

\begin{abstract}
Violence and aggression among patients suffering from mental health problems undoubtedly pose a challenge to healthcare professionals, families and carers. Aggressive behaviours affect all aspects of clinical care. The goal of professionals is to ensure safety while effectively managing behavioural emergencies. 'Rapid tranquillisation' implies prescribing pharmacological agents to manage these behaviours. This article highlights changing prescription trends. Appraisal of global guidelines suggests that factors other than scientific evidence dictate their evolution. Highquality randomised controlled trials are needed to develop a global guideline.
\end{abstract}

\section{The management of violence and aggression}

Violence and aggression among patients suffering from mental health problems undoubtedly pose a challenge to healthcare professionals. Families and carers find it difficult to cope and often are victims of the aggression. In-patient admission units may present a volatile environment where the surroundings can trigger or amplify such behaviours. The goal of healthcare professionals is to ensure safety while effectively managing behavioural emergencies.

Psychosis, concomitant physical illness and substance misuse or personality traits are frequently cited as aetiological factors leading to violence or aggression. In about $10 \%$ of these patients this may further escalate, leading to assaultive behaviour. Aggressive behaviours affect all aspects of clinical care. Apart from physical and psychological consequences, violent behaviour has considerable financial implications in terms of staff injuries and absenteeism (Hunter \& Carmel, 1992). A press release (National Health Service, 2005) stated that there were 43301 incidents of physical assaults against National Health Service staff working in mental health settings in 2004/05 across England.

Over the years, various interventions have been tried to manage violence and aggressive presentations. Blood-letting gained popularity after Paracelsus stated, 'Retention of toxic materials in the blood can lead to insanity'. Therapeutic bleeding was a panacea in ancient Egypt and Western cultures up to the late 1800s. Enemas and purgatives followed suit as an extension of this theory. Fasting and prayer were also used. Subsequently, the therapeutic effects of hypnotics were discovered and somnifacients were advocated.
More recently, guidelines advocate various non-pharmacological interventions, such as deescalation, before medications are used. They also recommend a holistic assessment replete with all relevant physical and laboratory investigations before the institution of pharmacotherapy (Expert Consensus Panel, 2005).

\section{Rapid tranquillisation}

It can sometimes be impossible to conduct a thorough physical examination of a violent patient. To ensure the safety of everyone involved, rapid tranquillisation of aggressive or violent patients may be unavoidable in these situations. Tranquillisation means calming without sedation. It is the use of psychotropic medication to control agitated, threatening or destructive psychotic behaviour, and is defined by the National Institute for Health and Care Excellence (NICE) as:

the use of medication to calm/lightly sedate the service user, reduce the risk to self and/or others and achieve an optimal reduction in agitation and aggression, thereby allowing a thorough psychiatric evaluation to take place, and allowing comprehension and response to spoken messages throughout the intervention. (NICE, 2005)

It is used when appropriate psychological and behavioural approaches have failed to de-escalate disturbed behaviour and is therefore essentially a last resort.

Historically, two strategies have been used for rapid tranquillisation:

- high-potency antipsychotic drugs, with an anxiolytic (benzodiazepine) or other drug for sedation

- low-potency antipsychotic drugs with both sedative and antipsychotic effects.

The procedure involves administering low doses of antipsychotic medication every 30-60 minutes with the specific goal of decreasing violence. A concurrent sedative medication often has the advantage of allowing a lower dose of antipsychotic, and therefore is associated with a decreased incidence of extrapyramidal side-effects. A lack of consensus among psychiatrists on the best medications to use for rapid tranquillisation highlights the need to have standardised guidelines which would enable some degree of uniformity in practice, although they would still remain only a guide and use would have to be tailored to individual patient needs, circumstances and available resources.

Interest has been rekindled in the topic by the inquiries into the deaths of patients Orville 
Blackwood in 1991 and David 'Rocky' Bennett in 1998 in the UK, both Afro-Caribbean males who died while in seclusion. They had always instilled a fear of violence in the nursing staff who had perhaps perceived them as 'big, black and dangerous'. The inquiry committees alerted psychiatrists to be wary of institutional bias against ethnic minorities owing to failure of Euro-centric psychiatry to appreciate cultural differences.

\section{The need for consensus and better guidelines}

A strong evidence base does not underpin rapid tranquillisation. Recommendations have therefore been based partly on research data and partly on clinical experience. One point of criticism is that studies have not included participants who were unable to consent.

The TREC Collaborative Group (2003) produced compelling evidence. Despite the robust methodology, the findings have not been incorporated into most guidelines. The 'zero tolerance' campaign of the UK National Health Service (NHS) has added another dimension to this issue. Most NHS trusts operate their own policies for rapid tranquillisation and emergency treatments.

There is no international consensus on the most effective treatment. Guidelines are statements of consensus and differ on which drugs to use. Clinical practice guidelines have been defined as 'systematically developed statements of recommendation for patient management to assist practitioner and patient decisions about appropriate health care for specific situations' (Institute of Medicine, 1990).

Clinician preference surveys also show variation. Antipsychotics and/or benzodiazepines are generally preferred as they can swiftly and safely calm patients. Given the scale of the problem there is a need to have a high-quality survey of clinician preferences and to critically examine the available evidence from guidelines recommending interventions for rapid tranquillisation.

\section{Appraisal of existing guidelines}

We identified seven national guidelines on rapid tranquillisation from five English-speaking countries: the UK, the USA, Canada, Australia and New Zealand (Nadkarni et al, 2014). Methodological quality was studied using the Appraisal of Guidelines Research and Evaluation (AGREE) instrument (AGREE Collaboration, 2003). All guidelines scored well on the domain of scope and purpose. The NICE guidelines consistently scored well on all domains with the exception of editorial independence. Surprisingly, the various guidelines lack uniformity in their recommendations. To cite examples, the guidelines from the Royal Australian and New Zealand College of Psychiatrists (RANZP) recommend benzodiazepines as the sole first-line option (McGorry, 2004). The NICE (2005) guidelines recommend using lorazepam only in cases of non-psychotic behavioural disturbance. The guidelines produced by the American
Psychiatric Association (APA, 2004) and those produced by the Canadian Psychiatric Association (CPA, 2005) recommend antipsychotics as the first line. The APA guidelines equate haloperidol with the atypical antipsychotics, whereas the CPA guidelines strictly recommend second-generation antipsychotics. The guidelines from the Royal Australian and New Zealand College of Psychiatrists (McGorry, 2004) recommend zuclopenthixol even in drug-naive patients, to avoid repeated injections (unlike the NICE and CPA guidelines).

\section{Clinician surveys and changing trends}

A search of the literature published between 1992 and 2005 revealed eight clinician practice surveys and two clinician preference surveys on rapid tranquillisation (Nadkarni et al, 2014). In 1994 most clinicians $(68 \%)$ opted for sedation as the desired outcome, while expecting it to be quick (Cunnane, 1994). In another survey, in $25 \%$ of incidents of aggression and violence patients received repeat doses of psychotropic agents within an hour of administration of the first agent (Pilowsky et al, 1992). Studies have found that higher initial doses do not lead to more rapid symptom resolution and are therefore unjustified for routine use (Huf et $a l, 2002)$. This was reflected in a local survey we conducted in an NHS hospital in 2009 (Nadkarni et al, 2014). From a total sample of 95 doctors, 60 (63\%) responded, of whom 51 (54\%) completed the questionnaires (nine were unable to complete the questionnaires owing to lack of experience, inadequate information or leaving employment). Of the respondents, $18 \%$ were consultants. As the drug of first choice, $45 \%$ would opt for lorazepam; other choices were olanzapine (33\%), haloperidol (12\%), haloperidol plus lorazepam $(6 \%)$ and haloperidol plus promethazine $(2 \%)$. The doses were within the range recommended by British National Formulary and choices conformed with the NICE guidelines. Preferences for monotherapy and the oral route (78\%) were in contrast to the older surveys; $92 \%$ aimed to achieve a calming effect without sedation, which was in keeping with the NICE guidelines.

\section{Final comments}

Clinician preferences have changed over time as recommendations from guidelines are incorporated in daily practice. This reflects the use of evidence-based medicine. What needs to be seen is how sound and unanimous this evidence is. It appears that the desired outcome and side-effects dictate the choice of the agent used for rapid tranquillisation. While a sedated patient might be the best solution in a busy, resource-stretched country such as Brazil, a calm patient is ideal in a higher-income country with adequate resources such as the USA or the UK. This may explain why guidelines differ in their choice of agents for rapid tranquillisation, although they access the same evidence pool. Despite there being a good-quality evidence base in the form of randomised controlled trials favouring promethazine (TREC Collaborative Group, 2003), it has not been licensed in the 
UK for use in rapid tranquillisation. This may be the result of these trials being conducted in a different country. Organisational and cultural differences between countries can lead to legitimate variations in recommendations. It is evident that treatments used for rapid tranquillisation still do not have a clear evidence base and uncertainty is still prevalent.

This work now raises a question: is current practice ethical in the UK, without the support of evidence from a well-designed randomised controlled trial? A local survey conducted in 2010 highlighted high conformity with NICE guidelines. However, it is evident that cultural and personal factors influence the recommendations not scientific evidence alone. Hence we conclude that high-quality randomised controlled trials with large samples are urgently needed. This will generate more evidence for the development of a global guideline rather than clinician preferences dictating their course. We can then hope to envisage evidence-based and ethical clinical practice in the near future.

\section{References}

AGREE Collaboration (2003) Development and validation of an international appraisal instrument for assessing the quality of clinical practice guidelines: the AGREE Project. Quality and Safety in Health Care, 12, 18-23.

APA (2004) Practice guideline for the treatment of patients with schizophrenia. Second edition. American Journal of Psychiatry, 161 (suppl. 2), 1-6.
CPA (2005) Clinical practice guidelines: treatment of schizophrenia. Canadian Journal of Psychiatry, 50 (suppl. 1), 7-57.

Cunnane, J. G. (1994) Drug management of disturbed behaviour by psychiatrists. Psychiatric Bulletin, 18, 138-139.

Expert Consensus Panel for Behavioural Emergencies (2005) Expert consensus guideline series: treatment of behavioural emergencies. Journal of Psychiatric Practice, 11 (suppl. 1), 5-108.

Huf, G., Coutinho, E., Fagundes, H., Jr, et al (2002) Current practices in managing acutely disturbed patients at three hospital in Rio de Janeiro, Brazil: a prevalence study. BMC Psychiatry, 2, 4.

Hunter, M. \& Carmel, H. (1992) The cost of staff injuries from inpatient violence. Hospital Community Psychiatry, 43, 586-588.

Institute of Medicine (1990) Clinical Practice Guidelines: Directions for a New Program. Institute of Medicine.

McGorry, P. (2004) Royal Australian and New Zealand College of Psychiatrists: clinical practice guidelines for the treatment of schizophrenia and related disorders. Australian and New Zealand Journal of Psychiatry, 39, 1-30.

Nadkarni, P., Jayaram, M., Nadkarni, S., et al (2014) Rapid tranquillisation - an AGREEable ground? Open Journal of Psychiatry, 4. 269-274.

National Health Service (2005) Press release: new measures to tackle violence against staff in mental health and learning disability services. NHS.

NICE (2005) Clinical Guideline 25. Violence: The Short-Term Management of Disturbed/Violent Behaviour in In-patient Psychiatric Settings and Emergency Departments. National Institute for Health and Care Excellence.

Pilowsky, L. S., Ring, H., Shine, P. J., et al (1992) Rapid tranquillisation: a survey of emergency prescribing in a general psychiatric hospital. British Journal of Psychiatry, 160, 831-835.

TREC Collaborative Group (2003) Rapid tranquillisation for agitated patients in emergency psychiatric rooms: a randomised trial of midazolam versus haloperidol plus promethazine. BMJ, 327 708-713.

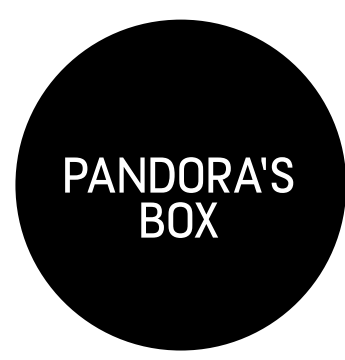

Pandora searches the world literature for evidence, news and other sources on matters of interest (doesn't shy away from controversy) to bring to the reader. She welcomes comments and suggestions (via ip@rcpsych. ac.uk)

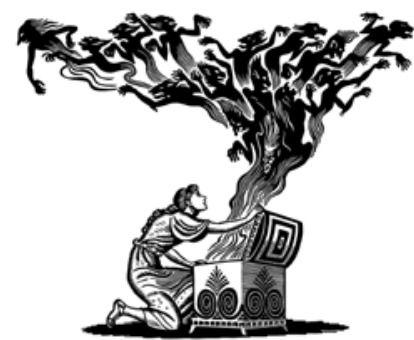

\section{World Suicide Prevention Day}

Tn 2003, 10 September was designated World 1 Suicide Prevention Day (WSPD), an annual event aiming to raise awareness and prompt action. The International Association for Suicide Prevention (IASP), the World Health Organization (WHO) and the World Federation for Mental Health (WFMH) collaborate to host WSPD. Suicide is a major social and public health issue! Nearly 1 million people around the world kill themselves every year. Every 40 seconds one person somewhere in the world puts an end to his or her life. Suicide is a global phenomenon. The highest rates are among those aged 70 or over, although globally suicide is the second leading cause of death among those 15-29 years old.

In September 2014 the WHO published its first 'World Suicide Report', Preventing Suicide: A Global Imperative, according to which $75 \%$ of suicides are in low- and middle-income countries. The WHO Director-General, Dr Chan, noted 'This report is a call for action to address a large public health problem which has been shrouded in taboo for far too long'.

The onset of these activities dates back to the 1990s, when concern about the high rates of suicide led some countries to approach the United Nations
(UN) and the WHO for help in designing national plans to tackle this problem in a cost-effective way. The UN, supported by the WHO, responded by issuing in 1996 the influential document 'Prevention of suicide: guidelines for the formulation and implementation of national strategies'. At that time only Finland had a government-sponsored initiative to develop a national framework and programme for suicide prevention, but within 15 years more than 25 low-, middle- and high-income countries had a strategy. In 2008, the WHO identified suicide as a priority condition in the Mental Health Gap Action Programme (mhGAP), designed to scale up care for mental, neurological and substance use disorders and particularly aimed at middle- and low-income countries. Research attention worldwide also turned to the prevention of suicide. WHO member states made a commitment to work towards a $10 \%$ reduction of suicide rates by the year 2020 . Time will show!

\section{A dream turned into a nightmare}

Continuing with the theme of suicide, the same USeptember 2014 WHO report states that 'while mental health problems play a role, which varies across different contexts, other factors, such as cultural and socio-economic status, are 